\title{
Evidências de Validade para o Teste de Retenção Visual de Benton (BVRT): Comparação de Grupos e Série de Casos em Pacientes Pós-AVC
}

\author{
Group Comparison and Case Series Studies in Stroke Patients: Evidence for Validity \\ of Benton Visual Retention Test (BVRT)
}

\author{
Joice Dickel Segabinazi ${ }^{1}$, Maxciel Zortea ${ }^{2}$, Jaqueline de Carvalho Rodrigues ${ }^{3}$, Juliana Burges Sbicigo ${ }^{4}$, \\ Clarissa Marceli Trentini ${ }^{5}$, Claudio Simon Hutz ${ }^{6}$, Denise Ruschel Bandeira ${ }^{7}$ \\ e Jerusa Fumagalli de Salles ${ }^{8}$
}

\begin{abstract}
Resumo
Investigaram-se evidências de validade de construto do Teste de Retenção Visual de Benton (BVRT) em pacientes após Acidente Vascular Cerebral (AVC). Participaram 42 adultos e idosos, $21 \mathrm{com}$ AVC (11 com Lesão em Hemisfério Esquerdo - LHE, 10 com Lesão em Hemisfério Direito - LHD), e 21 controles neurologicamente saudáveis, emparelhados aos pacientes por sexo, idade e anos de estudo. Foram aplicados o BVRT e tarefas de percepção visual, memória de trabalho, memória visual e praxia construtiva. Os grupos clínicos (LHE e LHD) apresentaram desempenho inferior na Administração C (Cópia) do BVRT. Na análise de série de casos, três casos com LHD e três casos com LHE demonstraram dissociações entre Administração A (Memória) e Administração C (Cópia) do teste. Os resultados apontaram que alguns pacientes pós-AVC, mesmo em fase crônica, podem apresentar déficits nas habilidades visuoconstrutivas e déficits mais leves em memória visual. Portanto, foram encontradas evidências de validade para o BVRT.
\end{abstract}

Palavras-chave: BVRT, memória visual, habilidades visuoconstrutivas, acidente vascular cerebral, validade

\begin{abstract}
Evidence for construct validity of the Visual Retention Test of Benton (BVRT) in patients after stroke was investigated. Participants were 42 adults and elderly, 21 with stroke (11 with Left Hemisphere Injury - LHE, 10 with Right Hemisphere Injury - LHD), and 21 neurologically healthy controls, paired to patients by sex, age and years of study. BVRT and tasks of visual perception, working memory, visual memory and constructive praxis were applied. The clinical groups (LHE and LHD) presented inferior performance in the Administration C (Copy) of the BVRT. In the case series analysis, three cases with LHD and three cases with LHE demonstrated dissociations between Administration A (Memory) and Administration C (Copy) of the test. The results showed that some post-stroke patients, even in the chronic phase, may present visuoconstructive skills deficits and lighter visual memory deficits. Therefore, evidence of validity for BVRT was found.
\end{abstract}

Keywords: BVRT, visual memory, visuoconstructive abilities, stroke, validity

\footnotetext{
${ }^{1}$ Doutora em Psicologia. Pontifícia Universidade Católica do Rio Grande do Sul (PUCRS). Faculdade de Psicologia. Professora Adjunta. Av Ipiranga, 6681, Prédio 11. Brasil. Tel.: 555133203500. Correo: jsegabinazi@ gmail.com

${ }_{2}^{2}$ Doutor em Psicologia. Hospital de Clínicas de Porto Alegre, Laboratório de Dor \& Neuromodulação. Pós-doutorado em andamento. Avenida Protásio Alves - até 1078, Porto Alegre, RS, Brasil. Tel.: 555133597604. Correo: max.zortea@gmail.com

${ }^{3}$ Doutora em Psicologia. Universidade do Vale do Rio dos Sinos. Professora Assistente. Centro de Ciências da Saúde. AC Unisinos, 950, Escola de Saúde, Brasil. Tel.: 555135911122. Correo: jaquecarvalhorodrigues@ gmail.com

${ }^{4}$ Doutora em Psicologia. Universidade Federal do Rio Grande do Sul (UFRGS). Instituto de Psicologia. Pós-doutorado em andamento. Av. Ramiro Barcelos, 2600, sala 214, Porto Alegre, RS, Brasil. Tel.: 555133085341. Correo: julianasbicigo@ gmail.com

${ }^{5}$ Doutora em Psicologia. Universidade Federal do Rio Grande do Sul (UFRGS). Instituto de Psicologia. Professor Associado. Av. Ramiro Barcelos, 2600, sala 219, Porto Alegre, RS, Brasil. Tel.: 555133085475. Correo: clarissatrentini@ terra.com.br

${ }^{6}$ Doutor em Psicologia. Universidade Federal do Rio Grande do Sul (UFRGS). Instituto de Psicologia. Professor Titular. Av. Ramiro Barcelos, 2600, sala 114, Porto Alegre, RS, Brasil. Tel.: 555133085246. Correo: claudio.hutz@ gmail.com

${ }^{7}$ Doutora em Psicologia. Universidade Federal do Rio Grande do Sul (UFRGS). Instituto de Psicologia. Professora Associada. Av. Ramiro Barcelos, 2600, sala 220, Porto Alegre, RS, Brasil. Tel.: 555133085352. Correo: deniserbandeira@gmail.com ${ }^{8}$ Doutora em Psicologia. Universidade Federal do Rio Grande do Sul (UFRGS). Professora Associada. Av. Ramiro Barcelos, 2600, sala 214, Porto Alegre, RS, Brasil. Tel.: 555133085341 Correo: jerusafsalles@gmail.com

Revista Iberoamericana de Diagnóstico y Evaluación - e Avaliação Psicológica. RIDEP · N54 · Vol.1 · 179-193 · 2020

ISSN: 1135-3848 print /2183-6051online
} 


\section{Introdução}

$\mathrm{Na}$ avaliação das funções visuoespaciais, as tarefas podem variar desde atividades mais simples, como o reconhecimento de objetos, até aquelas mais complexas que envolvem a integração de aspectos visuais, construtivos e executivos, como tarefas de visuoconstrução, incluindo a cópia de figuras (McCallum, 2017). O Teste de Retenção Visual de Benton (BVRT, Benton, 1945) é utilizado mundialmente para avaliação neuropsicológica da percepção, memória visual e habilidades visuoconstrutivas (Benton, 1945; Sivan, 1992). Na tarefa, o paciente deve reproduzir os estímulos memorizados (Administração A) ou que estejam à sua frente (Administração C) em uma folha de papel, sendo importante considerar a influência de aspectos perceptivos, motores, atentivos, executivos, linguísticos e motivacionais na execução do teste (Rodrigues, Duarte Junior, Czermainski, \& Salles, 2016). Durante sua realização, além de perceber as características visuoespaciais dos estímulos, a pessoa deve armazenar as figuras na memória visual de curto prazo, tarefa que possivelmente exige do componente visuoespacial da memória de trabalho (Baddeley, Allen, \& Hitch, 2011). Ainda, é plausível que a alça fonológica e o buffer episódico sejam recrutados, uma vez que é necessária a integração de informações semânticas e visuoespaciais já que muitos dos estímulos possuem figuras facilmente nomeáveis (Zanini et al., 2014).

Evidências de estudos utilizando Ressonância Magnética Funcional (fMRI) sugerem que os processos de memorizar e copiar figuras, exigidos pelo BVRT, estejam associados à ativação bilateral de áreas posteriores parietais (Gainotti \& Trojano, 2018), de regiões pré-motoras, frontais e temporais posteriores, além de áreas responsáveis pela linguagem (Potgieser, Hoorn, \& Jong, 2015). Essas evidências estão de acordo com os principais modelos cognitivo-neuropsicológicos (ex.: Harrington, Farias, \& Davis, 2009; Makuuchi, Kaminaga, \& Sugishita, 2003), nos quais a memória visual é considerada um processo cognitivo em que distintos componentes e regiões cerebrais são unificados para formar um todo coerente que exigem as mesmas regiões corticais associadas à percepção visual (Slotnick, 2004).
Estudos clínicos com adultos pós-Acidente Vascular Cerebral (AVC) mostraram que aqueles com Lesão de Hemisfério Direito (LHD) apresentaram um pior desempenho na Administração A do BVRT se comparados aos adultos com Lesão de Hemisfério Esquerdo (LHE) (Vakil, Blachstein, Sheleff, \& Groosman, 1989). Já pacientes com lesões vasculares exclusivamente subcorticais (núcleos da base e tálamo) mostraram déficit significativo na Administração A (Radanovic, Mansur, Azambuja, Porto, \& Scaff, 2004). Com relação à Administração C do BVRT, embora se acreditasse inicialmente que apenas um dos hemisférios cerebrais fosse responsável pelas habilidades visuoconstrutivas (Makuuchi et al., 2003), hoje são abundantes as evidências de que ambos os hemisférios estão envolvidos na execução dessa função (ex.: McCallum, 2017; Trojano \& Conson, 2008). Especificamente, há relatos de déficits nessas habilidades tanto em lesões parietais do hemisfério direito (em razão de um déficit na percepção espacial mais global), quanto do hemisfério esquerdo (relacionados a um déficit na representação das partes de um objeto) (Galeano \& Politis, 2008; Laeng, 2006).

Dois estudos brasileiros investigaram o perfil neuropsicolinguístico de pacientes que sofreram AVC (Fontoura, Rodrigues, Mansur, Monção, \& Salles, 2013; Pawlowski et al., 2013). No primeiro, 14 pacientes pós-AVC com LHE, com quadro de afasia expressiva, foram comparados a 16 controles. Os pacientes apresentavam características heterogêneas quanto ao tempo pósAVC e nível socioeconômico, e as lesões eram predominantemente nas regiões temporais, parietais e frontais. Além das esperadas dificuldades nas habilidades de linguagem expressiva, os pacientes apresentaram dificuldades em uma série de funções cognitivas, como as habilidades visuoconstrutivas (Fontoura et al., 2013). Por outro lado, 15 pacientes pós-AVC com LHE, sem quadro afásico, comparados a 30 controles (emparelhados por sexo, idade e escolaridade), apresentaram dificuldades em diversas tarefas de avaliação da linguagem, memória de trabalho e praxia ideomotora, mas não em tarefas de avaliação de memória visual de curto prazo e habilidades visuocontrutivas (Pawlowski et al., 2013). Portanto, dificuldades em memória visual e habilidades visuoconstrutivas em pacientes pós-AVC precisam 
ser investigadas na clínica neuropsicológica, pois estas capacidades estão relacionadas com a maioria das atividades de vida diária e sua redução pode trazer prejuízos funcionais importantes para esses pacientes (Nys et al., 2005a, 2005b).

A avaliação neuropsicológica deve ser pautada no uso de instrumentos com aporte neuropsicológico reconhecido e que apresentem evidências de validade de construto (ex.: Ayán et al., 2019; Pereira, Dias, Araújo, \& Seabra, 2018), principalmente relacionada ao critério. É importante investigar se o instrumento consegue determinar a existência de um prejuízo relacionado ao processo de resposta, ou seja, se o instrumento ajuda a compreender quais processos cognitivos subjazem o desempenho no instrumento (AERA, APA, \& NCME, 2014). Ao mesmo tempo, a identificação de funções neuropsicológicas deficitárias e preservadas auxilia na determinação do diagnóstico, prognóstico e na reabilitação do paciente pós-AVC (Pawlowski et al., 2013).

Assim, o objetivo geral deste trabalho foi obter evidências de validade de construto relacionada ao critério e ao processo de resposta para o BVRT em pacientes pós-AVC, incrementando a validade do teste em amostras brasileiras (Segabinazi et al., 2013; Zanini, Wagner, Salles, Bandeira, \& Trentini, 2012; Zanini et al., 2014). Foram objetivos específicos: 1) comparar os grupos (clínicos e controles) para obter evidências de validade de critério do BVRT, investigando também se haveria diferenças no desempenho da Administração A (Memória) e da Administração C (Cópia) com relação ao hemisfério cerebral afetado em pacientes com AVC (validade de critério); e 2) investigar o desempenho de adultos e idosos pósAVC, por meio de análise de série de casos, buscando evidências relacionadas ao processo de resposta do BVRT, comparando o desempenho no teste com outras medidas neuropsicológicas equivalentes e avaliando a existência de associações e dissociações entre os processos de memória visual e habilidades visuoconstrutivas.

\section{Método}

\section{Participantes}

Os adultos e idosos foram destros, brasileiros, monolíngues (falantes do Português Brasileiro), com no mínimo quatro anos de estudo formal. Os critérios de exclusão foram: a) história atual ou prévia de abuso de substâncias psicoativas (álcool e drogas ilícitas), b) distúrbios sensoriais (auditivos e/ou visuais não corrigidos) autorrelatados, c) dificuldades severas de compreensão da linguagem oral; c) presença de afasia (exceto afasia predominantemente expressiva), e d) presença de declínio cognitivo no grupo controle. Devido à alta frequência de pacientes com AVC que apresentam sintomas depressivos após o evento neurológico (ex.: Kouwenhoven, Kirkevold, Engedal, \& Kim, 2011), não foram excluídos pacientes que apresentaram depressão de moderada à grave. Os participantes dos dois grupos clínicos (LHE e LHD) apresentaram um episódio de AVC apenas em um hemisfério cerebral e não sofreram outros danos neurológicos, tais como tumor ou traumatismo cranioencefálico (confirmado por exames de imagem).

Após a análise dos critérios de exclusão, a amostra foi composta por 42 adultos (17 participantes com idade > 60): 11 casos com LHE, 10 casos com LHD, e 21 controles neurologicamente saudáveis, emparelhados aos pacientes por sexo, idade e anos de estudo, na proporção 1:1. Para cada paciente foi selecionado um controle de mesmo sexo, variando em, no máximo, dois anos de escolaridade e, no máximo, quatro anos de idade, para mais ou para menos, desde que pertencessem ao mesmo grupo (adultos ou idosos). Os controles de cada grupo clínico (controles dos casos com LHE e controles dos casos com LHD) não se diferenciaram entre si em relação às variáveis sexo, idade, escolaridade e hábitos de leitura e escrita. Assim, eles foram reunidos em um só grupo controle para algumas das análises. Igualmente, não foram encontradas diferenças estatisticamente significativas entre os grupos (clínicos e controles) para idade, anos de estudo e hábitos de leitura e escrita antes e após o AVC (Quadro 1). A caracterização sociodemográfica de cada caso do grupo clínico e do grupo controle é observada na Quadro 2. Os pacientes apresentaram um perfil heterogêneo de acordo com os tipos e locais de lesão (Quadro 3). 
Quadro 1. Dados Sociodemográficos e Demais Características dos Participantes por Grupo

\begin{tabular}{|c|c|c|c|c|c|}
\hline & \multicolumn{3}{|c|}{$\mathrm{M}(\mathrm{DP})$} & \multirow[b]{2}{*}{$F$} & \multirow[b]{2}{*}{$p$} \\
\hline & $\operatorname{LHE}(n=11)$ & LHD $(n=10)$ & Controles $(n=21)$ & & \\
\hline $\mathrm{Sexo} \mathrm{Fe} / \mathrm{M}(n)$ & $6 / 5$ & $6 / 4$ & $12 / 9$ & - & - \\
\hline Idade (anos) & $60.9(6.5)$ & $53.3(9.7)$ & $55.7(9.3)$ & 2.084 & .138 \\
\hline Anos de estudo & $8.6(4.1)$ & $9.3(3.5)$ & $9.3(3.5)$ & .229 & .796 \\
\hline Hábitos de L e E (pré) & $13.4(5.2)$ & $11.8(6.2)$ & $11.4(5.6)$ & .432 & .652 \\
\hline Hábitos de L e E (pós) & $9.4(5.8)$ & $9.1(4.4)$ & $11.4(5.6)$ & .875 & .425 \\
\hline QI Total & $94.3(15.4)$ & $94.9(12.7)$ & $100.7(8.45)$ & 1.503 & .235 \\
\hline Meses pós-AVC & $22.8(13.5)$ & $27.9(10.1)$ & - & .941 & .344 \\
\hline
\end{tabular}

Nota. Os valores de $F$ e $p$ foram gerados a partir de ANOVA de Um Fator. considerando os três grupos. LHE=lesão no hemisfério cerebral esquerdo; LHD=lesão no hemisfério cerebral direito; $\mathrm{AVC}=$ acidente vascular cerebral; $M=$ média; $D P=$ desvio-padrão; $\mathrm{Fe}=\mathrm{feminino;} \mathrm{M}=$ masculino; $\mathrm{L}=$ leitura; $\mathrm{E}=\mathrm{escrita}$; pré=hábitos de leitura e escrita antes do AVC; pós=hábitos de leitura e escrita após o AVC; Quociente Intelectual Total=QI calculada a partir de 4 subtestes da Escala de Inteligência de Wechsler Abreviada (WASI).

Quadro 2. Dados Sociodemográficos dos Participantes de Cada Grupo (LHE, LHD e Controles)

\begin{tabular}{|c|c|c|c|c|c|c|}
\hline Caso & Sexo & Idade (anos) & Estudo (anos) & Hábitos L e E ${ }^{\mathrm{a}}$ & Profissão & Classificação Econômicab \\
\hline LHE01 & $\mathrm{M}$ & 52 & 16 & Altos & Dona de casa & $\mathrm{A} 2$ \\
\hline LHE02 & M & 54 & 8 & Baixos & Manicure & $\mathrm{C} 1$ \\
\hline LHE03 & $\mathrm{F}$ & 56 & 10 & Baixos & Pedreiro & $\mathrm{C} 1$ \\
\hline LHE04 & $\mathrm{F}$ & 58 & 5 & Altos & Comerciante & $\mathrm{C} 1$ \\
\hline LHE05 & $\mathrm{F}$ & 59 & 15 & Altos & Laboratorista & $\mathrm{A} 2$ \\
\hline LHE06 & M & 61 & 11 & Baixos & Aux. Administrativo & B2 \\
\hline LHE07 & $\mathrm{F}$ & 61 & 9 & Baixos & Doméstica & $\mathrm{C} 1$ \\
\hline LHE08 & M & 60 & 5 & Baixos & Doméstica & B2 \\
\hline LHE09 & M & 66 & 8 & Altos & Professor & $\mathrm{C} 2$ \\
\hline LHE10 & $\mathrm{F}$ & 70 & 4 & Altos & Aux. Administrativo & $\mathrm{C} 1$ \\
\hline LHE11 & $\mathrm{F}$ & 73 & 4 & Baixos & Aux. Administrativo & $\mathrm{C} 2$ \\
\hline LHD12 & $\mathrm{F}$ & 58 & 5 & Baixos & Costureira & B2 \\
\hline LHD13 & M & 60 & 8 & Baixos & Motorista & B2 \\
\hline LHD14 & $\mathrm{F}$ & 61 & 4 & Baixos & Doméstica & $\mathrm{C} 2$ \\
\hline LHD15 & $\mathrm{F}$ & 67 & 11 & Baixos & Professora & B2 \\
\hline LHD16 & M & 38 & 14 & Baixos & Técnico contábil & B2 \\
\hline LHD17 & $\mathrm{F}$ & 37 & 11 & Baixos & Camareira & $\mathrm{C} 1$ \\
\hline LHD18 & $\mathrm{F}$ & 49 & 7 & Baixos & Dona de casa & $\mathrm{C} 1$ \\
\hline LHD19 & $\mathrm{F}$ & 51 & 14 & Altos & Aux. Administrativa & B1 \\
\hline LHD20 & M & 55 & 8 & Baixos & Frentista & $\mathrm{D}$ \\
\hline LHD21 & M & 57 & 11 & Altos & Vendedor & B1 \\
\hline $\mathrm{C} 01$ & M & 56 & 18 & Baixos & Professor & B2 \\
\hline $\mathrm{C} 02$ & M & 50 & 7 & Altos & Aux. Administrativo & B2 \\
\hline $\mathrm{C} 03$ & $\mathrm{~F}$ & 53 & 11 & Altos & Massoterapeuta & B2 \\
\hline $\mathrm{C} 04$ & $\mathrm{~F}$ & 54 & 6 & Baixos & Doméstica & B2 \\
\hline $\mathrm{C} 05$ & $\mathrm{~F}$ & 55 & 15 & Altos & Empresária & A2 \\
\hline $\mathrm{C} 06$ & M & 53 & 11 & Baixos & Mecânico & A2 \\
\hline $\mathrm{C} 07$ & $\mathrm{~F}$ & 63 & 8 & Baixos & Costureira & B2 \\
\hline $\mathrm{C} 08$ & M & 63 & 5 & Baixos & Segurança & $\mathrm{C} 2$ \\
\hline $\mathrm{C} 09$ & M & 66 & 8 & Baixos & Aux. Administrativo & B2 \\
\hline $\mathrm{C} 10$ & $\mathrm{~F}$ & 67 & 4 & Baixos & Costureira & B1 \\
\hline $\mathrm{C} 11$ & $\mathrm{~F}$ & 75 & 6 & Baixos & Secretária & $\mathrm{C} 1$ \\
\hline C12 & $\mathrm{F}$ & 59 & 8 & Altos & Jornalista & B2 \\
\hline $\mathrm{C} 13$ & M & 61 & 7 & Baixos & Aux. Construção civil & $\mathrm{C} 1$ \\
\hline $\mathrm{C} 14$ & $\mathrm{~F}$ & 60 & 5 & Baixos & Doméstica & B2 \\
\hline $\mathrm{C} 15$ & $\mathrm{~F}$ & 66 & 11 & Baixos & Dona de casa & B2 \\
\hline $\mathrm{C} 16$ & M & 40 & 15 & Altos & Aux. Administrativo & B2 \\
\hline $\mathrm{C} 17$ & $\mathrm{~F}$ & 36 & 13 & Baixos & Corretor de imóveis & $\mathrm{C} 2$ \\
\hline $\mathrm{C} 18$ & $\mathrm{~F}$ & 47 & 8 & Baixos & Dona de casa & $\mathrm{C} 1$ \\
\hline C19 & $\mathrm{F}$ & 53 & 15 & Baixos & Segurança & B1 \\
\hline $\mathrm{C} 20$ & M & 54 & 8 & Baixos & Porteiro & $\mathrm{C} 1$ \\
\hline $\mathrm{C} 21$ & M & 53 & 13 & Altos & Costureira & B1 \\
\hline
\end{tabular}

Nota. LHE=lesão no hemisfério cerebral esquerdo; LHD=lesão no hemisfério cerebral direito; C=controle; M=masculino; $\mathrm{F}=$ feminino; L=leitura; $\mathrm{E}=$ escrita; Aux=auxiliar. ${ }^{\mathrm{a}}$ Classificou-se como baixos hábitos de leitura e escrita um escore entre zero e 13 pontos e altos hábitos um escore entre 14 e 28 pontos no questionário sobre hábitos de leitura e escrita, descrito nos instrumentos (escore também proposto no estudo de Pawlowski et al.

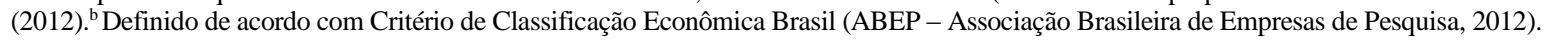


Quadro 3. Dados Neurológicos dos Grupos Clínicos (LHE e LHD)

\begin{tabular}{|c|c|c|c|c|c|}
\hline Caso & Etiologia & Região do AVC & Local da Lesão & Meses pós-AVC & BDI-II \\
\hline LHE01 & I & $S$ & $\begin{array}{c}\text { Cápsula interna, Globo pálido e } \\
\text { Tálamo }\end{array}$ & 21 & 4 \\
\hline LHE02 & $\mathrm{H}$ & Cs & Núcleos da base e parênquima & 14 & 21 \\
\hline LHE03 & I & S & Núcleos da base & 14 & 6 \\
\hline LHE04 & $\mathrm{H}$ & S & Núcleos da base & 28 & 12 \\
\hline LHE05 & I & $\mathrm{C}$ & Frontal & 17 & 23 \\
\hline LHE06 & I & S & Ínsula e Cápsula interna & 16 & 30 \\
\hline LHE07 & $\mathrm{H}$ & Cs & Parietal & 56 & 14 \\
\hline LHE08 & I & S & Coroa radiada & 36 & 15 \\
\hline LHE09 & I & Cs & Fronto-temporal & 18 & NA \\
\hline LHE10 & I & $S$ & Tálamo & 7 & 29 \\
\hline LHE11 & I & $S$ & Parieto-occiptal & 24 & NA \\
\hline LHD12 & I & Cs & Temporal & 48 & 23 \\
\hline LHD13 & I & $\mathrm{C}$ & Parietal & 24 & 24 \\
\hline LHD14 & $\mathrm{H}$ & $\mathrm{C}$ & Frontal & 22 & 11 \\
\hline LHD15 & I & $\mathrm{C}$ & Fronto-temporal & 26 & 15 \\
\hline LHD16 & I & Cs & Fronto-têmporo-parietal & 35 & 9 \\
\hline LHD17 & I & Cs & Frontal & 37 & 32 \\
\hline LHD18 & I & S & Núcleos da base & 24 & 17 \\
\hline LHD19 & $\mathrm{H}$ & $S$ & Coroa radiada e Núcleos da base & 29 & 23 \\
\hline LHD20 & $\mathrm{H}$ & Cs & Temporal & 23 & 4 \\
\hline LHD21 & I & Cs & Fronto-têmporo-parietal & 11 & 31 \\
\hline
\end{tabular}

Nota. LHE=lesão no hemisfério cerebral esquerdo; LHD=lesão no hemisfério cerebral direito; I=isquêmico; H= hemorrágico; C=Cortical; $\mathrm{S}=$ Subcortical; $\mathrm{Cs}=$ Cortico subcortical; NA=não se aplica, pois os pacientes responderam a GDS-15.

\section{Instrumentos e Procedimentos Específicos} Instrumentos para caracterização da amostra e verificação dos critérios de inclusão:

Questionário de condições de saúde e aspectos socioculturais (adaptado do estudo de Pawlowski, 2011): constam perguntas sobre dados pessoais, critérios de classificação econômica do Brasil (ABEP - Associação Brasileira de Empresas de Pesquisa, 2012), uso de substâncias lícitas e ilícitas, incluindo rastreio de problemas relacionados ao uso do álcool (Questionário CAGE - Ewing \& Rouse, 1970; versão de Amaral \& Malbergier, 2004), avaliação da dominância manual (Edinburgh Handedness Inventory Oldfield, 1971) e hábitos de leitura e escrita (Pawlowski et al., 2012). No questionário para os casos com AVC, foram acrescentadas perguntas sobre dados neurológicos dos participantes, como número de AVCs, tempo em meses, tipo e local de lesão.

Inventário de Depressão de Beck - BDI-II (Beck, Warrington, \& Brown, 1996; Gorestein, YuanPang, \& Arginon, 2011) e Escala de Depressão Geriátrica Yesavage - GDS-15 (Almeida \& Almeida, 1999; Yesavage et al., 1982): as escalas avaliam indícios de sintomas de depressão autorrelatados. $\mathrm{O}$ participante escolhia o item ou itens que melhor descrevessem como estava se sentindo na última

semana, incluindo o dia que estava respondendo ao teste. Dois participantes responderam a GDS15 e os demais responderam o BDI-II.

Mini Exame do Estado Mental (Folstein, Folstein, \& McHugh, 1975; versão adaptada por Chaves \& Izquierdo, 1992): instrumento do tipo screening utilizado para excluir da amostra casos controle com indícios de declínio cognitivo. Pessoas com baixa escolaridade deveriam apresentar escore $\leq 21$ e as com alta escolaridade deveriam apresentar escores $\geq 24 \mathrm{em}$ um total de 30 pontos (Kochhann, Varela, Lisboa, \& Chaves, 2010).

Token Test - versão reduzida (Fontanari, 1989; Moreira et al., 2011): foi utilizado nos grupos clínicos para avaliação da compreensão da linguagem oral, com 36 questões objetivas que aumentam em grau de complexidade. $\mathrm{O}$ participante deveria apontar ou movimentar círculos e quadrados de cores e tamanhos diferentes, conforme as ordens do examinador.

Teste de Boston para Diagnóstico das Afasias - versão reduzida (Goodglass, Kaplan, \& Barresi, 2001; Radanovic et al., 2004): instrumento utilizado para avaliar o diagnóstico de afasia, a partir das manifestações da fala e da linguagem 
que foi aplicado somente nos casos com LHE e em seus controles. Os subtestes avaliam no nível da palavra, da sentença e do discurso as seguintes habilidades: Conversação e Fala Espontânea, Compreensão Auditiva, Expressão Oral, Leitura e Escrita. O diagnóstico de afasia foi realizado por uma fonoaudióloga.

Instrumentos para avaliação neuropsicológica e cognitiva: Instrumento de Avaliação Neuropsicolinguística Breve para Afásicos Expressivos NEUPSILIN-Af (Fontoura, Rodrigues, Parente, Fonseca, \& Salles, 2011; Fontoura et al., 2013): trata-se de uma bateria breve, adaptada do Instrumento de Avaliação Neuropsicológica Breve NEUPSILIN (Fonseca, Salles, \& Parente, 2009) para avaliação neuropsicológica de pessoas com dificuldades expressivas de linguagem. Dessa bateria, foram analisadas somente as tarefas de Percepção Visual, Memória de Trabalho, Memória Visual e Praxia Construtiva.

Escala de Inteligência Wechsler Abreviada (WASI) (Wechsler Abbreviated Scale of Intelligence Manual - The Psychological Corporation, 1999; Trentini, Yates, \& Heck, 2014): aplicaram-se os subtestes Vocabulário, Semelhanças, Cubos e Raciocínio Matricial, que abrangem várias facetas da inteligência, tais como conhecimento verbal, processamento $\mathrm{e}$ informações visuais, raciocínio espacial e não verbal e inteligência cristalizada e fluida. Esses quatro subtestes compõem a Escala Completa e fornecem o QI Verbal (QIV), QI de Execução (QIE) e o QI Total (QIT). Os subtestes Vocabulário e Semelhanças compõem a Escala Verbal e fornecem o QI Verbal (QIV), e os subtestes Cubos e Raciocínio Matricial compõem a Escala de Execução e fornecem o QI de Execução (QIE). A medida de QIT foi covariável nas análises de comparações de grupos.

Teste de Retenção Visual de Benton (Benton Visual Retention Test - BVRT; Sivan, 1992; Salles, Bandeira, Trentini, Segabinazi, \& Hutz, 2016; Segabinazi et al., 2013): Foram utilizadas as combinações entre a Administração A (Forma C), para avaliação de memória visual, e Administração C (Forma D), para avaliação das habilidades visuoconstrutivas. Enquanto na Administração $\mathrm{A}$ as lâminas são apresentadas durante 10 segundos, na Administração $\mathrm{C}$ não há tempo limite de apresentação das lâminas, sendo que elas permanecem visíveis durante a execução do desenho. Em relação aos itens, cada uma das duas Formas possui 10 itens, sendo que os primeiros dois possuem apenas uma figura geométrica, e os outros oito consistem de duas figuras maiores e uma figura periférica menor. Os testes foram corrigidos por psicólogos treinados que participaram do processo de normatização do teste no Brasil e seguiram os critérios do manual brasileiro do teste (Salles et al., 2016). Para as análises foram computados os totais de erros e acertos tanto na Administração A (Forma C), quanto na Administração $\mathrm{C}$ (Forma D). Para melhor compreensão dos resultados e seguindo as normas propostas pelo Manual Brasileiro do BVRT (Salles et al., 2016) denominou-se a Administração A (Forma C) de Administração A (Memória) e a Administração C (Forma D) de Administração C (Cópia).

\section{Delineamento e Procedimentos Gerais}

$\mathrm{O}$ presente estudo possuiu um delineamento misto, quase-experimental de grupos contrastantes (LHE, LHD e controles) (Nachmias \& Nachmias, 1996), correlacional e de série de casos (Crawford, Garthwaite, \& Porter, 2010; Schwartz \& Dell, 2010). Selecionaram-se os participantes por amostragem não aleatória de conveniência. $\mathrm{O}$ estudo foi aprovado pelo Comitê de Ética em pesquisa do Instituto de Psicologia da Universidade Federal do Rio Grande do Sul (protocolo número 2009028) e do Hospital de Clínicas de Porto Alegre (HCPA), sob o número 100149.

Os participantes que apresentavam AVC foram contatados via telefone após indicação de médicos e residentes do Ambulatório de Neurovascular do Hospital de Clínicas de Porto Alegre (HCPA) ou após análise de seu prontuário neste hospital. Os participantes do grupo controle também foram contatados via telefone, a partir de indicações dos membros do projeto de pesquisa ou selecionados pelo banco de dados de servidores da UFRGS. Todos os participantes, ou seus responsáveis (no caso de pacientes), após concordarem em participar da pesquisa, assinaram o Termo de Consentimento Livre e Esclarecido (TCLE) e foram avaliados com os instrumentos para verificação dos critérios de exclusão. Foram realizados de dois a quatro 
encontros com cada um dos participantes, de aproximadamente 1 hora e 30 minutos de duração, ou até o momento que eles desejassem parar. Os encontros ocorriam na casa do participante, ou em salas da universidade, conforme sua preferência, ou quando este apresentava dificuldade de deslocamento.

\section{Análise de Dados}

\section{Comparações entre os grupos (LHE, LHD e controles)}

Realizaram-se análises descritivas (média, desvio-padrão e frequência) para delinear o perfil da amostra estudada (dados sociodemográficos e neurológicos). $\mathrm{Na}$ análise dos dados provenientes do BVRT, comparou-se o desempenho entre os três grupos (LHE, LHD e controles). Considerando-se as correlações moderadas entre a variável QI e os escores do BVRT (todas as correlações foram significativas a $p<.01 \quad \mathrm{e}$ conforme a direção esperada), optou-se por controlar essa variável nas comparações entre os grupos em análises de covariância (ANCOVA). Nas ANCOVAS, foram comparadas as variáveis: número de Acertos e Erros na Administração A (Memória) e Administração C (Cópia), escore Rasch de Memória e escore Rasch de Cópia. Os dois últimos escores foram calculados em um estudo anterior (Segabinazi et al., 2014) cujo objetivo foi aplicar a Teoria de Resposta ao Item na avaliação das propriedades psicométricas do BVRT e fornecer uma interpretação intervalar dos escores brutos no instrumento. Para isso, foram calculadas medidas logit correspondentes a cada escore bruto possível (soma dos itens corretos, variando de 0 a 10). Esse cálculo possibilita estimar a real posição de um indivíduo com um escore bruto x em um contínuo de habilidade, no caso do BVRT, nível de memória visual ou habilidades visuoconstrutivas.

\section{Análise de série de casos}

Utilizando a abordagem proposta por Schwartz e Dell (2010), primeiramente os casos do grupo clínico foram analisados quanto aos Acertos e Erros na Administração A (Memória), Acertos e Erros na Administração C (Cópia) e escores nas Tarefas de Percepção Visual, Memória de Trabalho, Memória Visual e Praxia Construtiva do NEUPSILIN-AF para testar a presença de desempenhos deficitários. Utilizou-se o método de Crawford, Garthwaite e Howell, (2009), o qual permite a comparação do caso com um grupo controle. Nesse método, o escore bruto do participante é transformado em escore $t$, o qual considera o tamanho amostral. Após a identificação dos casos com desempenho deficitário, foram investigadas dissociações entre Administração A (Memória) e Administração C (Cópia). Dissociações foram definidas pela classificação de Shallice (1988) e Crawford et al. (2009). Assim, dissociação clássica consiste em um caso que apresente escore deficitário (aqui foi considerado indicativo de déficit $[p<.10>.05])$, em uma das medidas e desempenho preservado na outra medida. A dissociação forte consiste em o caso apresentar escore deficitário em ambas as tarefas, porém ainda com uma diferença significativa indicando escore inferior em uma delas, em comparação a outra. Para tanto, utilizouse o teste para diferenças entre tarefas em casos que apresentam déficit em comparação a um grupo controle, conforme o cálculo proposto por Crawford et al. (2010), que considera a magnitude da correlação de Pearson entre os escores brutos das tarefas. Além disso, foram buscadas dissociações duplas, em que dois pacientes apresentam desempenhos diametralmente opostos, isto é, um caso mostra déficit na tarefa $\mathrm{X}$ e desempenho superior ou preservado na tarefa $\mathrm{Y}$, enquanto o outro caso apresenta déficit em $\mathrm{Y}$ e preservado ou superior em $\mathrm{X}$.

\section{Resultados}

\section{Comparações entre os grupos (LHE, LHD e controles)}

Nas comparações entre os três grupos não houve diferenças estatisticamente significativas nos escores de Acertos $[F(2,38)=1.34 ; p=.273] \mathrm{e}$ de Erros $[F(2,38)=1.46 ; \quad p=.244]$ na Administração A (Memória). Como as medidas Acertos e Erros na Administração C (Cópia), bem como nos escores Rasch de Memória e Cópia não apresentaram distribuição normal na amostra total, foram empregadas ANCOVAs não paramétricas, utilizando ranks e resíduos da Análise de Regressão (IBM Corp, 2012).

Realizaram-se análises ANOVAs de um fator, utilizando os resíduos estandardizados das regressões 
Quadro 4. Médias e Desvios Padrão dos Escores Brutos e Rasch do BVRT para os Grupos LHE. LHD e Controles

\begin{tabular}{lccc}
\hline & \multicolumn{3}{c}{ M (DP) } \\
\cline { 2 - 4 } & LHE $(n=11)$ & LHD $(n=10)$ & Controles $(n=21)$ \\
\hline Administração A (Memória) & & & \\
Acertos & $4.1(2.2)$ & $4.9(2.9)$ & $5.9(1.7)$ \\
Erros & $9.8(4.3)$ & $8.4(5.7)$ & $6.1(3.4)$ \\
Administração C (Cópia) & & \\
Acertos & $8.0(2.6)^{\mathrm{a}}$ & $7.5(3.4)^{\mathrm{a}}$ & $9.7(0.9)^{\mathrm{a} . \mathrm{b}}$ \\
Erros & $2.7(4.5)^{\mathrm{a}}$ & $3.3(4.2)^{\mathrm{a}}$ & $.3(9.6)^{\mathrm{a}}$ \\
Escore Rasch Memória & $-.6(1.8)$ & $1.9(1.9)$ & $.6(1.2)$ \\
Escore Rasch Cópia & $2.1(1.8)^{\mathrm{a}}$ & $.0(2.3)^{\mathrm{a}}$ & $3.4(0.9)^{\mathrm{a} . \mathrm{b}}$ \\
\hline
\end{tabular}

Nota. ${ }^{\text {a.b }}=$ letras diferentes indicam, conforme teste Tukey, diferenças significativas no nível de $p<.05$.

Quadro 5. Desempenhos nas Tarefas do NEUPSILIN-AF para os Casos que Apresentaram Associação, Dissociação Forte e Dissociação Clássica nos Escores do BVRT

\begin{tabular}{|c|c|c|c|c|c|c|}
\hline \multirow[b]{2}{*}{ Caso } & \multicolumn{6}{|c|}{ NEUPSILIN-AF } \\
\hline & Percepção visual & $\begin{array}{l}\text { Memória de } \\
\text { trabalho }\end{array}$ & Memória visual & $\begin{array}{c}\text { Praxia } \\
\text { construtiva }\end{array}$ & $\begin{array}{c}\text { Administração A } \\
\text { (Memória) }\end{array}$ & $\begin{array}{c}\text { Administração C } \\
\text { (Cópia) }\end{array}$ \\
\hline \multicolumn{7}{|c|}{ Casos que apresentaram dissociações fortes } \\
\hline LHD14 & Preservada & Preservada & Preservada & Déficit & Déficit & Déficit \\
\hline LHD20 & Preservada & Déficit & Déficit & Déficit & Déficit & Déficit \\
\hline LHE04 & $\begin{array}{l}\text { Sugestivo de } \\
\text { déficit }\end{array}$ & Déficit & Déficit & Déficit & Déficit & Déficit \\
\hline LHE11 & Preservada & Preservada & Déficit & Déficit & $\begin{array}{c}\text { Sugestivo de } \\
\text { déficit }\end{array}$ & Déficit \\
\hline \multicolumn{7}{|c|}{ esentaram disso } \\
\hline LHD17 & Preservada & Preservada & Preservada & Preservada & Preservada & Sugestivo de défici \\
\hline LHE06 & Preservada & Preservada & Preservada & Preservada & Preservada & Sugestivo de défici \\
\hline
\end{tabular}

Nota. Para identificação de déficits, foi utilizada a transformação dos escores brutos em escores $t$ sugerida por Crawford et al. (2009).

Essa transformação permite comparar o escore do paciente com o do grupo controle, levando em consideração o tamanho amostral reduzido. Para graus de liberdade $=20$, escores $t$ menores que $-1,725(p<.05)$ indicaram déficit (escores $t$ entre $-1,725 \mathrm{e}-1,325$ foram considerados sugestivos de déficit $[.10>p \geq .05])$.

entre as medidas do BVRT e os escores de QI. Os resultados evidenciaram diferenças entre os três grupos (LHE, LHD e controles) para Acertos na Administração $C$ (Cópia) $[F(2,39)=4.93$; $p=.012]$, Erros na Administração C (Cópia) [ $F(2$, $39)=5.18 ; p=.010]$ e escores Rasch de Cópia $[F(2$, 39)=4.93; $p=.012$ ], porém os escores Rasch para Memória não foram diferentes entre os grupos $[F$ $(2,39)=1.82 ; p=.175]$. Testes post - hoc de Tukey indicaram que tanto o grupo LHD $(p=.043)$ quanto LHE $(p=.033)$ tiveram menores escores de Acertos de Cópia do que o grupo controle. O mesmo padrão ocorreu no caso do escore Rasch de Cópia, tanto para pacientes LHD $(p=.045)$ quando LHE $(p=.031)$. Conforme esperado, na variável escore de Erros na Administração $C$ (Cópia), os pacientes com LHD apresentaram maior escore de erros $(p=.030)$, assim como pacientes com LHE ( $p=.034$ ), em comparação aos controles. Os grupos de pacientes LHE e LHD não se diferenciaram entre si em nenhuma das medidas fornecidas pelo BVRT, porém apresentaram desempenhos significativamente distintos dos controles.

\section{Séries de casos}

Foram encontrados seis casos com perfil deficitário no BVRT, sendo três pacientes do grupo LHD (LHD14, LHD17 e LHD20) e três do grupo LHE (LHE04, LHE06 e LHE11), os quais demonstraram dissociações entre Administração A (Memória) e Administração C (Cópia), conforme Quadro 5. Os desempenhos nas tarefas do NEUPSILIN-Af foram utilizados para descrição detalhada do perfil neuropsicológico destes casos (Quadro 5).

Quatro casos apresentaram dissociações fortes (LHD14, LHD20, LHE04, LHE11) com déficit (ou indicativo de déficit, sendo que para o caso LHE11, o valor de $p=.058$ ) nos escores de Acertos na Administração A (Memória) e Administração C (Cópia), conforme Figura 1a. Contudo, os escores de Acertos na Administração C (Cópia) foram significativamente inferiores aos de Acertos na Administração A (Memória), configurando, assim, uma dissociação forte. Igualmente, esses pacientes apresentaram prejuízos significativos nos Erros na Administração A (Memória) e Administração C (Cópia), ainda que os de Erros 
a)

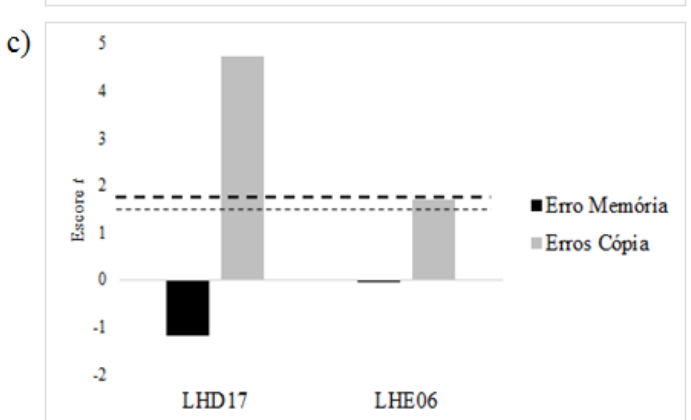

b)

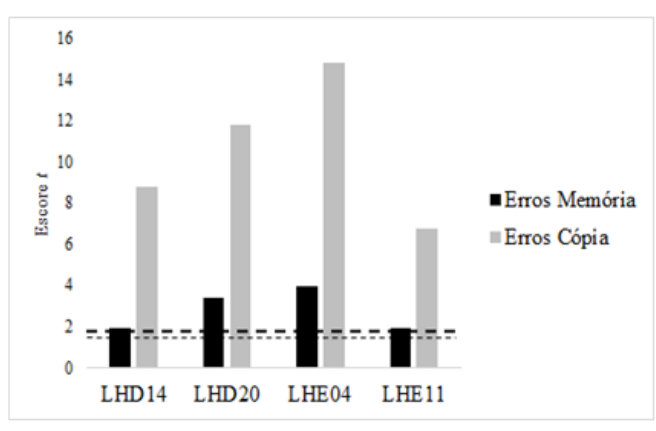

d)

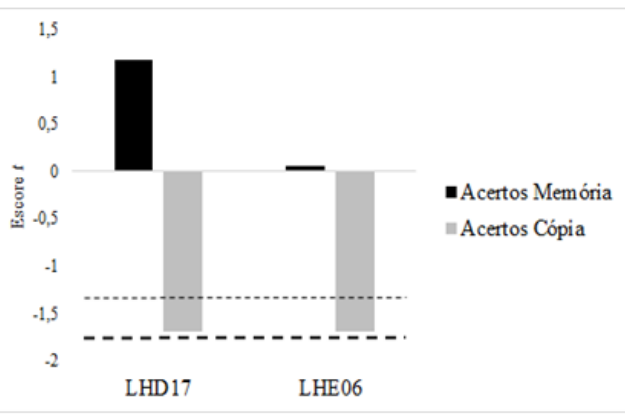

Figura 1. Associações e Dissociações nos casos clínicos. segundo o desempenho no BVRT. a) casos que mostraram dissociações fortes para Acertos na Administração A (Memória) e Administração C (Cópia); b) casos que mostraram dissociações fortes para Erros na Administração A (Memória) e Administração C (Cópia); c) casos que mostraram dissociações clássicas para Acertos na Administração A (Memória) e Administração C (Cópia) e d) casos que mostraram dissociações clássicas para Erros na Administração A (Memória) e Administração C (Cópia). As linhas tracejadas indicam o limite de $p=.10$ e $p=.05$.

na Administração C (Cópia) sejam significativamente maiores do que os de Erros na Administração C (Memória), para todos os casos, conforme Figura $1 \mathrm{~b}$.

Dois casos apresentaram dissociações clássicas: LHD17 e LHE06 (Figura 1c). Para estes casos, o escore de Acertos na Administração C (Cópia) foi significativamente inferior ao escore de Acertos na Administração A (Memória). Além disso, o escore de Acertos na Administração C (Cópia) foi sugestivo de déficit ( $p=.054$ para ambos os casos), porém o escore de Acertos na Administração A (Memória) foi dentro do esperado, conforme comparação com grupo controle. $\mathrm{Na}$ análise de erros no BVRT, o paciente LHD17 apresentou déficit e o paciente LHE06 mostrou escore sugestivo de déficit ( $p=.054)$ em Erros na Administração C (Cópia), porém os escores foram significativamente inferiores aos de Erros na Administração A (Memória), este se mantendo dentro do esperado (Figura 1d). Não foram encontrados casos que apresentassem dissociação dupla.

\section{Discussão}

Este estudo investigou evidências de validade de construto do BVRT através da comparação do desempenho de grupos de pacientes pós-AVC e da análise de série de casos. Inicialmente, compararam-se os grupos (LHE e LHD) para procurar evidências de validade de critério do BVRT investigando diferenças no desempenho da Administração A (Memória) e Administração C (Cópia) com relação ao hemisfério afetado em pacientes com AVC. Nesse aspecto, não foram encontradas diferenças significativas entre os grupos clínicos (LHE e LHD). Esse resultado não pôde confirmar uma hipótese de especialização hemisférica para as habilidades de memória visual e habilidades visuocontrutivas, como alguns autores sugerem (Laeng, 2006; Galeano \& Politis, 2008; Trojano \& Conson, 2008). Entretanto, em função da grande variabilidade de locais de lesão entre os participantes e do tamanho amostral, não descartamos a possibilidade de diferenças de desempenho caso fosse estudada uma amostra mais homogênea (e mais ampla) quanto a características do AVC. É ainda importante salientar que tanto o funcionamento do hemisfério cerebral direito quanto do esquerdo são exigidos na realização de tarefas visuoconstrutivas. De acordo com Lezak et al. (2012), lesões cerebrais à esquerda podem estar relacionadas com dificuldades com detalhes de um desenho, mesmo que o todo ainda esteja correto.

A comparação do desempenho dos grupos 
clínicos com o grupo controle evidenciou escores inferiores para os grupos clínicos no BVRT somente na Administração C (Cópia) e no escore Rasch de Cópia, controlando-se o nível de QI dos participantes. Dessa maneira, pode-se afirmar que as diferenças entre os grupos não poderiam ser atribuídas ao rebaixamento cognitivo geral, mas sim a dificuldades nas habilidades visuoconstrutivas. Déficits significativos na memória visual e nas habilidades visuoconstrutivas de pacientes pós-AVC são mais frequentemente encontrados em grupos de pacientes com lesões cerebrais agudas (Nys et al., 2005a). A pesquisa realizada por Nys et al. (2005a) encontrou que em seis a dez meses pósAVC, cerca de $83 \%$ dos pacientes já apresentam uma recuperação significativa nas capacidades de visuoconstrução e $78 \%$ na capacidade de memória visual. A amostra investigada no presente estudo reuniu pacientes que tinham, em média, 25 meses pós-AVC, tempo superior ao dos estudos com pacientes pós-AVC que encontraram déficits de memória visual (Wilde, 2006). Assim, é plausível que déficits na memória visual tenham ocorrido na fase aguda do AVC, porém já tivessem sido compensados na época da avaliação deste estudo ou que déficits na memória visual nunca tenham ocorrido. Ainda, a análise de grupos não busca avaliar a variabilidade intra-grupo. De fato, foram encontrados casos com desempenho de memória visual rebaixado, porém apenas na análise de série de casos, conforme será discutido a seguir. Estudos futuros com o BVRT poderão investigar amostras pós-AVC em fase aguda, a fim de verificar as singularidades nos escores do BVRT (Acertos, Erros e Tipos de erros) em fases inicias pós-lesão.

A análise de série de casos foi utilizada para buscar evidências relacionadas ao processo de resposta do BVRT. A estratégia de análise de série de casos utilizada neste estudo e desenvolvida por Crawford et al. (2010) avança no método de estudos de caso em Neuropsicologia ao considerar critérios estatísticos para indicar perfis de associação e dissociação. Destaca-se que seis casos apresentaram dissociações entre os escores de memória e cópia do BVRT, sendo que quatro casos apresentaram dissociações fortes. $\mathrm{Ou}$ seja, os pacientes demonstraram déficits significativamente maiores na Administração A
(Memória) quando comparados com a Administração C (Cópia) e dois apresentaram dissociações clássicas, ou seja, déficits significativos na Administração C (Cópia), mas não na Administração A (Memória). Assim, a observação de dissociações fortes e clássicas entre a Administração A (Memória) e a Administração C (Cópia) do BVRT reforça a utilidade do instrumento na determinação de prejuízos na percepção visual, memória visual e habilidades visuoconstrutivas em pacientes pós-AVC (Sivan, 1992; Salles et al., 2016). Em se tratando de um quadro tão heterogêneo quanto o de pacientes pósAVC, é importante que não apenas sejam analisados os pacientes em grupo, mas também com uma abordagem de análise de desempenho de casos.

$\mathrm{Na}$ análise de série de casos, examinaram-se adicionalmente os desempenhos dos pacientes nas tarefas do NEUPSILIN-Af (Fontoura et al., 2011), observando-se um padrão de potencialidades e prejuízos nessas funções. Em quatro casos nos quais foram encontradas dissociações fortes, dois deles apresentaram, além de déficits no escore de Acertos e Erros da Administração C (Cópia) do BVRT, prejuízos em Memória de Trabalho verbal do NEUPSILIN-Af. Baddeley e Hitch (1994) descreveram o componente visuoespacial como um dos componentes do modelo de Memória de Trabalho, sendo responsável pela manipulação da informação visual. Assim, os casos LHD20 e LHE04 podem ter apresentado dificuldade na Administração C (Cópia), também por apresentar um déficit de Memória de Trabalho, o qual é exigido durante as tarefas que envolvem planejamento e execução espacial. Essa hipótese precisa ser melhor investigada, uma vez que as tarefas de Memória de Trabalho do Neupsilin-Af são apresentadas apenas na modalidade verbal. $\mathrm{O}$ caso LHE04 também apresentou déficits no escore de Percepção Visual, podendo indicar dificuldades específicas na relação com o estímulo e sua percepção, o que pode ter comprometido a reprodução dos estímulos do BVRT na Cópia (Guérin et al., 1999).

Por meio dos escores no NEUPSILIN-Af foi possível observar que todos os pacientes apresentaram déficits significativos na tarefa de Praxia Construtiva (Cópia). Essa tarefa consiste em itens que variam em complexidade (desde a 
cópia de um quadrado até o desenho de um relógio com números e ponteiros em um horário específico) (Fontoura et al., 2011). Esse achado e o fato de todos os pacientes apresentarem desempenho prejudicado nos escores de Acertos e Erros Administração C (Cópia) do BVRT incrementam as evidências de validade relacionada ao critério do BVRT.

Além disso, a presença de dificuldades nas habilidades visuoconstrutivas, ainda que a amostra tenha sido composta por pacientes com um tempo médio pós-lesão de 25 meses destaca a importância de intervenções direcionadas a recuperação dessas funções em paciente pósAVC, mesmo na fase crônica. Em relação aos padrões específicos de desempenho de acordo com os tipos de lesões (Corticais, Subcorticais e Cortico subcorticais) ou os locais de lesão, não foi possível observar relações da mesma maneira que foi encontrado em outro estudo (Rodrigues, Pawlowski, Zibetti, Fonseca, \& Parente, 2011). Ainda que tenham sido buscadas relações entre variáveis sociodemográficas e o desempenho dos casos que apresentaram dissociações, não foi possível determinar um padrão, dada a grande variabilidade da amostra nesta variável. Entretanto, os resultados aqui relatados reforçam as indicações de que dificuldades nas habilidades visuoconstrutivas podem ocorrer em razão de lesões em diferentes regiões e em decorrência dos distintos processamentos cognitivos que são necessários para essa tarefa. Assim, é plausível que as manifestações neuropsicológicas geradas a partir de uma lesão cerebral não sejam decorrentes apenas da área cerebral específica danificada, mas também das conexões desta área com as demais regiões cerebrais (Trojano \& Conson, 2008).

Com relação às bases neurais subjacentes às tarefas de memorização de estímulos visuais e à cópia desses estímulos, tal como o BVRT avalia, ressalta-se que se trata de habilidades altamente complexas e compostas por múltiplos componentes cognitivos. O emprego de técnicas de neuroimagem vem possibilitando um mapeamento sistemático das relações entre estruturas cerebrais e funções neuropsicológicas (Trojano, Grossi, \& Flash, 2009). Acredita-se que apenas as inovações tecnológicas expressas pelas diferentes técnicas de avaliação online das funções cognitivas e técnicas de neuroimagem, aliadas ao método anatomoclínico, os estudos de caso único e as pesquisas que incluem uma série de casos possam permitir um conhecimento maior sobre o papel de determinadas áreas cerebrais, além de outras funções relacionadas às habilidades visuoconstrutivas e à memória visual. Dessa forma, futuros estudos com o BVRT deverão lançar mão dessas técnicas para determinar, por exemplo, padrões de ativação cerebral e de movimentos oculares durante a realização do teste.

\section{Considerações Finais}

Os resultados deste estudo forneceram novas evidências de validade do BVRT em amostras brasileiras (Salles et al., 2016; Zanini et al., 2012; Zanini et al., 2014) através de um método misto de investigação, no qual foi possível detectar déficits no desempenho pós-AVC. Esses déficits foram observados na comparação de grupos principalmente nas habilidades visuoconstrutivas, Administração C (Cópia) do BVRT, e na comparação de série de casos na memória visual, Administração A (Memória). Ainda, a constatação de dissociações fortes e clássicas traz implicações práticas e teóricas à Neuropsicologia, indicando a utilidade do BVRT à avaliação clínica de déficits dessas funções separadamente, o que pode subsidiar o planejamento de programas de reabilitação do paciente.

O presente estudo possui algumas limitações. A seleção da amostra foi realizada por conveniência, assim não se podem generalizar os resultados. Outro aspecto é o extenso intervalo de tempo entre a avaliação neurológica (neuroimagem anatômica) e a avaliação clínica, de modo que possa ter existido a recuperação das funções neuropsicológicas avaliadas pelo BVRT em alguns dos pacientes. Além disso, não foram encontradas informações precisas sobre a participação dos indivíduos que compuseram o grupo clínico em programas de reabilitação. Os resultados apontaram que, pacientes pós-AVC, mesmo em fase crônica ainda podem apresentar déficits importantes nas habilidades visuoconstrutivas. Este achado é relevante se considerarmos que as intervenções focadas na recuperação dessas funções são frequentemente citadas em estudos (ex.: Suzuki et al., 2013) como ponto de partida da reabilitação, sendo 
determinante para predizer a recuperação da funcionalidade pós-AVC.

\section{Referências}

ABEP - Associação Brasileira de Empresas de Pesquisa. (2012). Critério de classificação econômica. Recuperado em 1 de Junho de 2018 , de http://www.abep.org/novo/Content.aspx ?Cont entID=301

Almeida, O. P., \& Almeida, S. A. (1999). Confiabilidade da versão brasileira da Escala de Depressão em Geriatria (GDS) - versão reduzida. Arquivos de Neuro-Psiquiatria, 57(2B), 421-426. doi:10.1590/S0004-282X1999000300013

Amaral, R. A. do, \& Malbergier, A. (2004). Avaliação de instrumento de detecção de problemas relacionados ao uso do álcool (CAGE) entre trabalhadores da prefeitura do campus da Universidade de São Paulo (USP) campus capital. Revista Brasileira de Psiquiatria, 26(3). doi:10.1590/S1516-44462004000300005

American Educational Research Association, American Psychological Association, and National Council on Measurement in Education (2014). Standards for Educational and Psychological Testing. Washington, DC.

Ayán, C., Cancela, J. M., Sánchez-Lastra, M. A., Carballo-Roales, A. I., Domínguez-Meis, F., \& Redondo-Gutiérrez, L. (2019). Fiabilidad y validez de la Batería TGMD-2 en población española. Revista Iberoamericana de Diagnóstico y Evaluación - e Avaliação Psicológica, $\quad 50(1) \quad 21-33$. doi:10.21865/RIDEP50.1.02

Baddeley, A. D., \& Hitch, G. J. (1994). Developments in the concept of working memory. Neuropsychology, 8(4), 485-493. doi:10.1037/0894-4105.8.4.485

Baddeley, A. D., Allen, R. J., \& Hitch, G. J. (2011). Binding in visual working memory: The role of the episodic buffer. Neuropsychologia, 49(6), 1393-1400. doi:10.1016/j.neuropsychologia.2010.12.042

Beck, A. T., Warrington, E. K., \& Brown, G. (1996). BDI-II: Beck Depression Inventory-II
Manual. New York, NY: The Psychological Corporation.

Benton, A. L. (1945). A visual retention test for clinical use. Archives of Neurology and Psychiatry, 52, 2012-2016. doi:10.1001/archneurpsyc.1945.0230009005100 8

Sivan, A. B (1992). Benton Visual Retention Test. Manual (5th ed.). USA: The Psychological Corporation.

BRASIL. (1996). Lei no 9334, de 20 de dezembro de 1996. Estabelece as diretrizes e bases da educação nacional. Brasília: Diário Oficial Da União, 134(248).

Chaves, M. L., \& Izquierdo, I. (1992). Differential diagnosis between dementia and depression: A study of efficiency increment. Acta Neurologica Scandinavica, 85(6), 378-382. doi:10.1111/j.1600-0404.1992.tb06032.x

Crawford, J. R., Garthwaite, P. H., \& Howell, D. C. (2009). On comparing a single case with a control sample: An alternative perspective. Neuropsychologia, 47(13), 2690-2695. doi:10.1016/j.neuropsychologia.2009.04.011

Crawford, J. R., Garthwaite, P. H., \& Porter, S. (2010). Point and interval estimates of effect sizes for the case-controls design in neuropsychology: Rationale, methods, implementations, and proposed reporting standards. Cognitive Neuropsychology, 27(3), 245-260. doi:10.1080/02643294.2010.513967

Ewing, J. A., \& Rouse, B. A. (1970). Identifying the hidden alcoholic. Presented at the 29th International Congress on Alcohol and Drug Dependence, Sidney, Australia.

Folstein, M. F., Folstein, S. E., \& McHugh, P. R. (1975). "Mini-mental state": A practical method for grading the cognitive state of patients for the clinician. Journal of Psychiatry Resources, 12, 189-198. doi:10.1016/0022-3956(75)90026-6

Fonseca, R. P., Salles, J. F., \& Parente, M. A. M. P. (2009). Instrumento de Avaliação Neuropsicológica Breve Neupsilin. São Paulo: Vetor.

Fontanari, J. L. (1989). O Token Test: Elegância e concisão na avaliação da compreensão do afásico. Validação da versão reduzida de De Renzi para o português. Neurobiologia, 52(3), 177-218.doi:10.1590/S0101-60832011000300003 
Fontoura, D. R., Rodrigues, J. C., Mansur, L., Monção, A. M., \& Salles, J. F. (2013). Neuropsycholinguistic profile of patients poststroke in the left hemisphere with expressive aphasia. Revista Neuropsicología, Neuropsiquiatría y Neurociencias, 13(2), 91110.

Fontoura, D. R., Rodrigues, J. C., Parente, M. A. M. P., Fonseca, R. P., \& Salles, J. F. (2011). Adaptação do Instrumento de Avaliação Neuropsicológica Breve NEUPSILIN para avaliar pacientes com afasia expressiva: NEUPSILIN-Af. Ciências \& Cognição, 16(3), 78-94. Recuperado em 01 de junho de 2018 , de

http://pepsic.bvsalud.org/pdf/cc/v16n3/v16n3a 08.pdf

Gainotti, G., \& Trojano, A. L. (2018). Constructional apraxia. In G. Vallar \& H. B. Coslett, Handbook of Clinial Neurology (v.151-3ed series, pp. 331-348). Amsterdam: Elsevier.

Galeano, A. R., \& Politis, D. G. (2008). Trastornos visuoconstructivos. In E. Labos (Ed.), Tratado de Neuropsicología Clínica (p bp. 253-257). Buenos Aires: Librería Akadia.

Goodglass, H., Kaplan, E., \& Barresi, B. (2001). Boston Diagnostic Aphasia Examination Short Form. Philadelphia, USA: Lippincott Williams \& Wilkins.

Gorestein, C., YuanPang, W., Arginon, I. L. A., \& Werlang, B. S. G. (2011). Manual do Inventário de Depressão de Beck - BDI-II. São Paulo: Casa do Psicólogo.

Guérin, F., Ska, B., \& Belleville, S. (1999). Cognitive processing of drawing abilities. Brain and Cognition, 40(3), 464-478. doi:10.1006/brcg.1999.1079

Harrington, G. S., Farias, D., \& Davis, C. H. (2009). The neural basis for simulated drawing and the semantic implications. Cortex, 45(3), 386-393. doi:10.1016/j.cortex.2007.10.015

IBM Corp. (2012). Can SPSS do a nonparametric or rank analysis of covariance (Quade's test)? Retrieved from http://www01.ibm.com/support/docview.wss?uid=swg21 477497

Kochhann, R., Varela, J. S., Lisboa, C. S. M., \& Chaves, M. L. (2010). The Mini Mental State
Examination: Review of cutoff points adjusted for schooling in a large Southern Brazilian sample. Dementia \& Neuropsychologia, 4(1), 35-41. doi:10.1590/S1980-57642010DN40100006

Kouwenhoven, S. E., Kirkevold, M., Engedal, K., \& Kim, H. S. (2011). Depression in acute stroke: Prevalence, dominant symptoms and associated factors. A systematic literature review. Disability and Rehabilitation, 33(7), 539-556. doi:10.3109/09638288.2010.505997

Laeng, B. (2006). Constructional apraxia after left or right unilateral stroke. Neuropsychologia, 44(9), 1595-1606. doi:10.1016/j.neuropsychologia.2006.01.023

Lezak, M. D., Howieson, D. B., Bigler, E. D., \& Tranel, D. (2012). Neuropsychological assessment (5a ed.). New York: Oxford University Press.

McCallum, S. (2017). Handbook of nonverbal assessment. Cham, Switzerland: Springer International Publishing

Makuuchi, M., Kaminaga, T., \& Sugishita, M. (2003). Both parietal lobes are involved in drawing: A functional MRI study and implications for constructional apraxia. Brain Research. Cognitive Brain Research, 16(3), 338-347. doi:10.1016/S0926-6410(02)00302-6

Moreira, L., Schlottfeldt, C. G., Paula, J. J. de, Daniel, M. T., Paiva, A., Cazita, V., Coutinho, G., Salgado, J. V., \& Malloy-Diniz, L. F. (2011). Estudo normativo do Token Test versão reduzida: Dados preliminares para uma população de idosos brasileiros. Revista de Psiquiatria Clínica, 38(3), 97-101. doi:10.1590/S0101-60832011000300003

Nachmias, C., \& Nachmias, D. (1996). Research methods in the social sciences. London, UK: Arnold.

Nys, G. M. S., van Zandvoort, M. J. E., De Kort, P. L. M., Jansen, B. P. W., Van Der Worp, H. B., Kappelle, L. J., \& De Haan, E. H. F. (2005a). Domain-specific cognitive recovery after first-ever stroke: A follow-up study of 111 cases. Journal of the International Neuropsychological Society, 11(07). doi:10.1017/S1355617705050952

Nys, G. M. S., van Zandvoort, M. J. E., de Kort, P. L. M., van der Worp, H. B., Jansen, B. P. W., Algra, A., Haan, E. H. F., \& Kappelle, L. 
J. (2005b). The prognostic value of domainspecific cognitive abilities in acute first-ever stroke. Neurology, 64(5), 821-827. doi:10.1212/01.WNL.0000152984.28420.5A

Oldfield, R. C. (1971). The assessment and analysis of handedness: The Edinburgh inventory. Neuropsychologia, 9(1), 97-113. doi:10.1016/0028-3932(71)90067-4

Pawlowski, J. (2011). Instrumento de Avaliação Psicológica Breve NEUPSILIN: Evidências de validade de construto $e$ de validade incremental à avaliação neuropsicológica (Tese de Doutorado). Universidade Federal do Rio Grande do Sul, Porto Alegre, Brasil.

Pawlowski, J., Remor, E., Parente, M. A. M. P., Salles, J. F., Fonseca, R. P., \& Bandeira, D. R. (2012). The influence of reading and writing habits associated with education on the neuropsychological performance of Brazilian adults. Reading and Writing, 25(9), 22752289. doi:10.1007/s11145-012-9357-8

Pawlowski, J., Rodrigues, J. C., Martins, S. C. O., Brondani, R., Chaves, M. L., Fonseca, R. P., \& Bandeira, D. R. (2013). Avaliação neuropsicológica breve de adultos pósacidente vascular cerebral em hemisfério esquerdo. Avances En Psicología Latinoamericana/Bogotá (Colombia), 31(1), 3345. Recuperado em 01 de junho de 2018, de http://www.scielo.org.co/pdf/apl/v31n1/v31n1a 03.pdf

Pereira, A. P. P., Dias, N. M., Araújo, A. M., \& Seabra, A. G. (2018). Funções executivas na infância: Avaliação e dados normativos preliminares para crianças portuguesas em idade pré-escolar. Revista Iberoamericana de Diagnóstico y Evaluación - e Avaliação Psicológica, $\quad$ 49(4), 171-188. doi:doi.org/10.21865/RIDEP49.4.14

Potgieser, A. R. E, van der Hoorn, A., \& de Jong, B. M. (2015). Cerebral activations related to writing and drawing with each hand. PLoS ONE, 10(5), e0126723. doi:10.1371/journal.pone.0126723

Radanovic, M., Mansur, L. L., \& Scaff, M. (2004). Normative data for the Brazilian population in the Boston Diagnostic Aphasia Examination: Influence of schooling. Brazilian Journal of Medical and Biological
Research, $37(11)$, $1731-1738$ doi:10.1590/S0100-879X2004001100019

Rodrigues, J. C., Duarte Junior, S., Czermainski, F. R., \& Salles, J. F. (2016). O Teste de Retenção Visual de Benton, funções neuropsicológicas investigadas e indicações para uso em neuropsicologia. In J. F. Salles, Bandeira, D. R., Trentini, C., Segabinazi, J., \& Hutz, C. (Eds.), Manual do Teste de Retenção Visual de Benton (pp.17-26). São Paulo: Vetor.

Rodrigues, J. C., Pawlowski, J., Zibetti, M. R., Fonseca, R. P., \& Parente, M. A. M. P. (2011). Avaliação de apraxias em pacientes com lesão cerebrovascular no hemisfério esquerdo. Psicologia: Teoria E Pesquisa, 13(2), 209-220. Recuperado em 01 de junho de 2018, de http://editorarevistas.mackenzie.br/index.php/ ptp/article/view/3761

Salles, J. F., Bandeira, D. R., Trentini, C. M., Segabinazi, J. D., \& Hutz, C. S. (2016). Manual do Teste de Retenção Visual de Benton: Manual profissional. Editora: Vetor.

Schwartz, M. F., \& Dell, G. S. (2010). Case series investigations in cognitive neuropsychology. Cognitive Neuropsychology, 27(6), 477-494. doi:10.1080/02643294.2011.574111

Segabinazi, J. D., Junior, S. D., Salles, J. F., Bandeira, D. R., Trentini, C. M., \& Hutz, C. S. (2013). Teste de Retenção Visual de Benton: Apresentação do manual brasileiro. Avaliação Psicológica, 12, 421428. Recuperado em 01 de junho de 2018 , de http://pepsic.bvsalud.org/pdf/avp/v12n3/v12n 3a18.pdf

Segabinazi, J. D., Yates, D. B., Salles, J. F., Trentini, C. M., Hutz, C. S., \& Bandeira, D. R. (2014). Aplicação do modelo de Rasch na avaliação das propriedades psicométricas do Teste de Retenção Visual de Benton. Manuscrito em preparação.

Shallice, T. (1988). From neuropsychology to mental structure. London: Cambridge University Press.

Slotnick, S. D. (2004). Visual memory and visual perception recruit common neural substrates. Behavioral and Cognitive Neuroscience Reviews, 3(4), 207-221. doi:10.1177/1534582304274070 
Suzuki, M., Sugimura, Y., Yamada, S., Omori, Y., Miyamoto, M., \& Yamamoto, J. (2013). Predicting recovery of cognitive function soon after stroke: Differential modeling of logarithmic and linear regression. PLOS ONE, $8(1)$ e53488. doi:10.1371/journal.pone.0053488

The Psychological Corporation. (1999). Wechsler Abbreviated Scale of Intelligence Manual. San Antonio: APA.

Trentini, C. M., Yates, D. B., \& Heck, V. S. (2014). Escala de Inteligência Wechsler Abreviada (WASI): Manual Profissional. São Paulo: Pearson.

Trojano, L., \& Conson, M. (2008). Visuospatial and visuoconstructive deficits. Handbook of Clinical Neurology, 88, 373-391. doi:10.1016/S0072-9752(07)88019-5

Vakil, E., Blachstein, H., Sheleff, P., \& Groosman, S. (1989). BVRT - scoring system and time delay in the differentiation of lateralized hemispheric damage. International Journal of Clinical Neuropsychology, 11(3), 125-128. Recuperado em 1 de Junho de 2018, de https://faculty.biu.ac.il/ vakil/papers/Vaki_19 89_(3).pdf

Yesavage, J. A., Brink, T. L., Rose, T. L., Lum, O., Huang, V., Adey, M., \& Leirer, V. O. (1982). Development and validation of a geriatric depression screening scale: A preliminary report. Journal of Psychiatric Research, 17(1), 37-49. doi:10.1016/0022-3956(82)90033-4

Zanini, A. M., Wagner, G. P., Salles, J. F. de, Bandeira, D. R., \& Trentini, C. M. (2012). Teste de Retenção Visual de Benton (BVRT): Evidências de validade para idosos. Avaliação Psicológica, 11(2), 287-296. Recuperado em 01 de junho de 2018, de http://pepsic.bvsalud.org/pdf/avp/v11n2/v11n 2a14.pdf

Zanini, A., Wagner, G., Zortea, M., Segabinazi, J., Salles, J., Bandeira, D., \& Trentini, C. (2014). Evidence of criterion validity for the Benton Visual Retention Test: Comparison between older adults with and without a possible diagnosis of Alzheimer's disease. Psychology and Neuroscience, 7(2), 131-138. doi:10.3922/j.psns.2014.006
Wilde, M. C. (2006). The validity of the repeatable battery of neuropsychological status in acute stroke. The Clinical Neuropsychologist, 20(4), 702-715. doi:10.1080/13854040500246901 